\title{
TROPICAL MATRIX GROUPS
}

\author{
ZUR IZHAKIAN $^{1}$, MARIANNE JOHNSON ${ }^{2}$ and MARK KAMBITES ${ }^{3}$
}

\begin{abstract}
We study the subgroup structure of the semigroup of real square matrices of given dimension under tropical matrix multiplication. We show that every maximal subgroup is isomorphic to the full linear automorphism group of a related tropical polytope, and that each of these groups is the direct product of $\mathbb{R}$ with a finite group. We also show that there is a natural and canonical embedding of each full rank maximal subgroup into the group of units of the semigroup. Out results have numerous corollaries, including the fact that every automorphism of a full rank projective tropical polytope extends to an automorphism of the containing space, and that every full rank subgroup has a common eigenvector.
\end{abstract}

\section{INTRODUCTION}

Tropical algebra is the algebra of the real numbers (sometimes augmented with an extra element denoted by $-\infty$ ) under the operations of addition and maximum. It has applications in areas such as combinatorial optimisation and scheduling, control theory, and algebraic geometry to name but a few (see [3] for a survey of applications). Many problems arising from these application areas are naturally expressed using (tropical) linear equations, so much of tropical algebra concerns matrices.

In this paper, we study the semigroup of real $n \times n$ square matrices with tropical multiplication. A important step in understanding tropical algebra is to understand the maximal subgroups of the semigroup, in terms of both their abstract group structure and the geometric of their natural actions on tropical space. It is a basic fact of semigroup theory that every subgroup of a semigroup $S$ lies in a unique maximal subgroup. Moreover, the maximal subgroups of $S$ are precisely the $\mathcal{H}$-classes (see Section 3 below for definitions) of $S$ which contain idempotents element. Recent research of the authors into the structure of idempotent matrices [10] provides a useful basis for studying subgroups.

In addition to this introduction, this article comprises seven sections. In Section 2 we introduce some preliminary definitions. In Section 3 we give a brief account of Green's relations for the semigroup of $n \times n$ tropical matrices and prove that every maximal subgroup $H$ is isomorphic to the

\footnotetext{
${ }^{1}$ Fachbereich Mathematik, Universät Bremen, D-28359 Bremen, Germany. Email zzur.zzur@gmail.com. Research supported by the Alexander von Humboldt Foundation.

${ }^{2}$ School of Mathematics, University of Manchester, Manchester M13 9PL, England. Email Marianne.Johnson@maths.manchester.ac.uk. Research supported by EPSRC Grant EP/H000801/1.

${ }^{3}$ School of Mathematics, University of Manchester, Manchester M13 9PL, England. Email Mark.Kambites@manchester.ac.uk. Research supported by EPSRC Grant $\mathrm{EP} / \mathrm{H} 000801 / 1$. Mark Kambites gratefully acknowledges the hospitality of Universität Bremen during a visit to Bremen.
} 
automorphism group of a particular tropical polytope (namely, the column space of any element of $H$ ). In Sections 4 and 5 we summarise a number of results on idempotent tropical matrices.

A consequence of [10] is that there is an extremely well-behaved notion of rank for tropical idempotents, and hence for maximal subgroups of tropical matrices. The understanding of idempotents arising from [10] is markedly more comprehensive when the idempotents in question have full rank. Accordingly, Section 6 reduces the problem of understand maximal subgroups to the full rank case, by showing that every maximal subgroup of $n \times n$ matrices of rank $k<n$ is naturally isomorphic to a maximal subgroup of $k \times k$ matrices.

Finally, Section 7 establishes our strongest results. We exhibit a natural and canonical embedding of each full rank maximal subgroup (and hence of each subgroup) into the group of units of the semigroup. An analysis of this embedding allows us to show that each maximal subgroup of rank $k$ is the direct product of $\mathbb{R}$ with a finite group of permutation degree $k$ or less.

The decomposition of maximal subgroups of direct products of $\mathbb{R}$ with finite groups establishes in the finitary case a conjecture of the second and third authors [12], which states that every group of $n \times n$ tropical matrices has a torsion free abelian subgroup of index $n$ ! or less. This conjecture has been independently proved in the general case by Shitov [14]. It is natural to ask exactly which exactly which finite groups arise in these decompositions. In a paper of the the second and third authors [13], we shall show that for every finite group $G$, the group $G \times \mathbb{R}$ arises as a maximal subgroup in sufficiently high dimensions.

Of course, it is also natural to ask about the subgroup structure of the full semigroup of $n$ timesn matrices over the larger tropical semiring including $-\infty$. Indeed, there are already a few interesting results in this direction $[12,14]$. In this article we have chosen to focus on matrices with finite entries, partly to avoid technicalities and partly because some of the machinery and results we employed have not yet been developed for the case with $-\infty$. However, we believe the results and methods developed here should, with careful application, suffice to permit a full understanding of the subgroup structure in the more general case.

Other corollaries of our results include that every automorphism of a projective tropical polytope extends to an automorphism of the containing space, and that every subgroup has a common eigenvector.

\section{Preliminaries}

We write $\mathbb{F} \mathbb{T}$ for the set $\mathbb{R}$ equipped with the operations of maximum (denoted by $\oplus$ ) and addition (denoted by $\otimes$, by + or simply by juxtaposition). Thus, we write $a \oplus b=\max (a, b)$ and $a \otimes b=a b=a+b$. It is readily verified that $\mathbb{F} \mathbb{T}$ is an abelian group (with neutral element 0 ) under $\otimes$ and a commutative semigroup of idempotents (without a neutral element) under $\oplus$, and that $\otimes$ distributes over $\oplus$. These properties mean $\mathbb{F} \mathbb{T}$ has the structure of an idempotent semifield.

It will sometimes be convenient to work with the extended tropical semifield $\mathbb{T}=\mathbb{F} \mathbb{T} \cup\{-\infty\}$, where we extend the definitions of $\oplus$ and $\otimes$ in the 
obvious way (namely, $a \oplus-\infty=-\infty \oplus a=a$ and $a \otimes-\infty=-\infty \otimes a=-\infty$, for all $a \in \mathbb{T})$.

Let $M_{n}(\mathbb{F} \mathbb{T})$ denote the set of all $n \times n$ matrices with entries in $\mathbb{F} \mathbb{T}$. The operations $\oplus$ and $\otimes$ can be extended in the obvious way to give corresponding operations on $M_{n}(\mathbb{F} \mathbb{T})$. In particular, it is easy to see that $M_{n}(\mathbb{F} \mathbb{T})$ is a semigroup with respect to tropical matrix multiplication. We shall see in the following sections that this semigroup has a rich and interesting structure.

We shall be interested in the space $\mathbb{F}^{n}$ consisting of $n$-tuples $x$ with entries in $\mathbb{F} \mathbb{T}$; we write $x_{i}$ for the $i$ th component of $x$. We call $\mathbb{F}^{n}$ (affine) tropical $n$-space. The space $\mathbb{F}^{n}$ admits an addition and a scaling action of $\mathbb{F} \mathbb{T}$ given by $(x \oplus y)_{i}=x_{i} \oplus y_{i}$ and $(\lambda x)_{i}=\lambda\left(x_{i}\right)$ respectively. These operations give $\mathbb{F}^{n}$ the structure of an $\mathbb{F} \mathbb{T}$-module $e^{4}$. It also has the structure of a lattice, under the partial order given by $x \leq y$ if $x_{i} \leq y_{i}$ for all $i$.

From affine tropical $n$-space we obtain projective tropical $(n-1)$-space, denoted $\mathbb{P F} \mathbb{T}^{n-1}$, by identifying two vectors if one is a tropical multiple of the other by an element of $\mathbb{F} \mathbb{T}$. We identify $\mathbb{P F} \mathbb{T}^{n-1}$ with $\mathbb{R}^{n-1}$ via the map

$$
\left(x_{1}, \ldots, x_{n}\right) \mapsto\left(x_{1}-x_{n}, x_{2}-x_{n}, \ldots, x_{n-1}-x_{n}\right) .
$$

Submodules of $\mathbb{F}^{n}$ (that is, subsets closed under tropical addition and scaling) are termed (tropical) convex sets. Finitely generated convex sets are called (tropical) polytopes. Since convex sets are closed under scaling, each convex set $X \subseteq \mathbb{F T}^{n}$ induces a subset of $\mathbb{P F} \mathbb{T}^{n-1}$, termed the projectivisation of $X$ and denoted $\mathcal{P} X$.

For $A \in M_{n}(\mathbb{F} \mathbb{T})$ we let $R(A)$ denote the tropical polytope in $\mathbb{F}^{n}$ generated by the rows of $A$ and let $C(A)$ denote the tropical polytope in $\mathbb{F}^{n}$ generated by the columns of $A$. We call these tropical polytopes the row space and column space of $A$ respectively.

A point $x$ in a convex set $X$ is called extremal in $X$ if the set

$$
X \backslash\{\lambda \otimes x: \lambda \in \mathbb{F} \mathbb{T}\}
$$

is a submodule of $X$. Clearly some scaling of every such extremal point must lie in every generating set for $X$. In fact, every tropical polytope is generated by its extremal points considered up to scaling $[4,15]$.

\section{Green's Relations, idempotents And REgularity}

Green's relations are five equivalence relations $(\mathcal{L}, \mathcal{R}, \mathcal{H}, \mathcal{D}$ and $\mathcal{J})$ and three partial orders $\left(\leq_{\mathcal{R}}, \leq_{\mathcal{L}}\right.$ and $\left.\leq_{\mathcal{J}}\right)$, which can be defined on any semigroup, and which describe the structure of its maximal subgroups and principal left, right and two-sided ideals. We briefly recap the definitions here; for further details (including proofs of the claimed properties) we refer the reader to an introductory text such as [9].

Let $S$ be any semigroup. If $S$ is a monoid, we set $S^{1}=S$, and otherwise we denote by $S^{1}$ the monoid obtained by adjoining a new identity element 1 to $S$. We define a binary relation $\leq_{\mathcal{R}}$ on $S$ by $a \leq_{\mathcal{R}} b$ if $a S^{1} \subseteq b S^{1}$, that is, if either $a=b$ or there exists $q$ with $a=b q$. We define another relation $\mathcal{R}$ by $a \mathcal{R} b$ if and only if $a S^{1}=b S^{1}$. It is straight-forward to check that $\mathcal{R}$ is

\footnotetext{
${ }^{4}$ Some authors use the term semimodule, to emphasise the non-invertibility of addition, but since no other kind of module exists over $\mathbb{F} \mathbb{T}$ we have preferred the more concise term.
} 
an equivalence relation, and $\leq_{\mathcal{R}}$ is a preorder (a reflexive, transitive binary relation) which induces a partial order on the $\mathcal{R}$-equivalence classes.

The relations $\leq_{\mathcal{L}}$ and $\mathcal{L}$ are the left-right duals of $\leq_{\mathcal{R}}$ and $\mathcal{R}$ (that is, $a \leq_{\mathcal{L}} b$ if $S^{1} a \subseteq S^{1} b$, and $a \mathcal{L} b$ if $\left.S^{1} a=S^{1} b\right)$. The relations $\leq_{\mathcal{J}}$ and $\mathcal{J}$ are two-sided analogues $\left(a \leq \mathcal{J} b\right.$ if $S^{1} a S^{1} \subseteq S^{1} b S^{1}$, and $a \mathcal{J} b$ if $S^{1} a S^{1}=S^{1} b S^{1}$ ). The relations $\mathcal{H}$ and $\mathcal{D}$ are described in terms of the $\mathcal{L}$ and $\mathcal{R}$ relations. The $\mathcal{H}$ relation is the intersection of $\mathcal{L}$ and $\mathcal{R}$ (that is, $a \mathcal{H} b$ if $a \mathcal{L} b$ and $a \mathcal{R} b$ ), whilst the $\mathcal{D}$ relation can be defined by $a \mathcal{D} b$ if and only if there exists an element $c \in S$ such that $a \mathcal{R} c$ and $c \mathcal{L} a$. It can be shown that both $\mathcal{H}$ and $\mathcal{D}$ are equivalence relations.

The study of Green's relations for the full tropical matrix semigroups was initiated (in the case of $M_{2}(\mathbb{T})$ ) by the second and third authors [12]. In [8], Hollings and the third author gave a complete description of the $\mathcal{D}$-relation for $M_{n}(\mathbb{F} \mathbb{T})$, using the duality between the row and column space of a tropical matrix. In [11] the second and third authors described the equivalence relation $\mathcal{J}$ and pre-order $\leq_{\mathcal{J}}$ in in $M_{n}(\mathbb{F} \mathbb{T})$ and $M_{n}(\mathbb{T})$. The main results of these papers, for the case $M_{n}(\mathbb{F} T)$, are summarised in the following theorem (see [8, Proposition 3.1 and Theorem 5.1] and [11, Theorem 5.3 and Theorem 6.1] for full details and proofs).

Theorem 3.1. Let $A, B \in M_{n}(\mathbb{F} \mathbb{T})$.

(i) $A \leq_{\mathcal{L}} B$ if and only if $R(A) \subseteq R(B)$;

(ii) $A \mathcal{L} B$ if and only if $R(A)=R(B)$;

(iii) $A \leq_{\mathcal{R}} B$ if and only if $C(A) \subseteq C(B)$;

(iv) $A \mathcal{R} B$ if and only if $C(A)=C(B)$;

(v) $A \mathcal{H} B$ if and only if $R(A)=R(B)$ and $C(A)=C(B)$;

(vi) $A \mathcal{D} B$ if and only if $C(A)$ and $C(B)$ are isomorphic as $\mathbb{F} \mathbb{T}$-modules;

(vii) $A \mathcal{D} B$ if and only if $R(A)$ and $R(B)$ are isomorphic as $\mathbb{F} \mathbb{T}$-modules;

(viii) $A \leq \mathcal{J} B$ if and only if there exists a convex set $X \subseteq \mathbb{F}^{n}$ such that $R(A)$ embeds linearly into $X$ and $R(B)$ surjects linearly onto $X$;

(ix) $A \leq \mathcal{J} B$ if and only if there exists a convex set $X \subseteq \mathbb{F}^{n}$ such that $C(A)$ embeds linearly into $X$ and $C(B)$ surjects linearly onto $X$.

(x) $A \mathcal{J} B$ if and only if $A \mathcal{D} B$.

Parts (i)-(v) of the above theorem are straight-forward, whilst parts (vi)(x) require considerably more work (the proofs make use of the row-column duality alluded to above as well as some elementary topological arguments).

The following result follows immediately from the definitions.

Lemma 3.2. Let $A, B \in M_{n}(\mathbb{F} \mathbb{T})$.

(i) If $A \leq_{\mathcal{L}} B$ then any linear relation between the columns of $B$ induces the same relation between the columns of $A$.

(ii) If $A \leq_{\mathcal{R}} B$ then any linear relation between the rows of $B$ induces the same relation between the rows of $A$.

Proof. We prove (i), the proof of (ii) being dual. Let $A \leq_{\mathcal{L}} B$. If $A=B$, the result holds trivially. Assume then that $A=X B$ for some $X \in M_{n}(\mathbb{F} T)$. Define $f: C(B) \rightarrow C(A)$ to be left multiplication by $X$. Thus $f$ is a linear map sending the $i$ th column of $B$ to the $i$ th column of $A$ and it follows that any relation between the columns of $B$ induces the corresponding relation between the columns of $A$. 
In fact, there is a correspondence between inclusions of row spaces and certain surjections of column spaces; see [8] for full details.

Theorem 3.3. [8, Theorem 4.2, Corollary4.3].

(i) $R(A) \subseteq R(B)$ if and only if there there is a surjective linear morphism from $C(B)$ to $C(A)$ taking the ith column of $B$ to the ith column of $A$ for all $i$.

(ii) $R(A)=R(B)$ if and only if there is a linear isomorphism from $C(A)$ to $C(B)$ taking the ith column of $B$ to the ith column of $A$ for all $i$.

(iii) $C(A) \subseteq C(B)$ if and only if there there is a surjective linear morphism from $R(B)$ to $R(A)$ taking the ith row of $B$ to the ith row of $A$ for all $i$.

(iv) $C(A)=C(B)$ if and only if there is a linear isomorphism from $R(A)$ to $R(B)$ taking the ith row of $B$ to the ith row of $A$ for all $i$.

We shall require a few more semigroup theoretic definitions. Let $S$ be any semigroup. We recall that $e \in S$ is an idempotent if $e^{2}=e$, whilst $a \in S$ is (von Neumann) regular if there exists $x \in S$ with $a x a=a$. It is easy to see that $a$ is regular if and only if $a$ is $\mathcal{R}$-related to an idempotent (dually, if and only if $a$ is $\mathcal{L}$-related to an idempotent). Moreover, it is well known that every subgroup of a semigroup $S$ lies in a unique maximal subgroup, and that the maximal subgroups are precisely the $\mathcal{H}$-classes of $S$ containing idempotents.

A complete description of the idempotent elements of $M_{2}(\mathbb{F} \mathbb{T})$ was given in [12] and it follows from the results given there that the semigroup $M_{2}(\mathbb{F} \mathbb{T})$ is regular (that is, every $\mathcal{R}$ class and every $\mathcal{L}$ class contains an idempotent) and each maximal subgroup is isomorphic to either $\mathbb{R}$ or $\mathbb{R} \times S_{2}$. For $n \geq 3$ it is known that the semigroups $M_{n}(\mathbb{F} \mathbb{T})$ are no longer regular. In [10] the present authors gave a geometric characterisation of the regular elements of $M_{n}(\mathbb{F} \mathbb{T})$. In the present paper we turn our attention to the maximal subgroups of $M_{n}(\mathbb{F} \mathbb{T})$, in other words, the $\mathcal{H}$-classes containing an idempotent tropical matrix. Given an idempotent tropical matrix $E$, we first note that the $\mathcal{H}$ class containing $E$ is isomorphic to the group of $\mathbb{F} \mathbb{T}$-module automorphisms of $C(E)$.

Theorem 3.4. Let $E$ be an idempotent in $M_{n}(\mathbb{F} \mathbb{T})$, with corresponding $\mathcal{H}$ class of denoted by $H_{E}$, and let $\operatorname{Aut}(C(E))$ denote the group of $\mathbb{F} \mathbb{T}$-module automorphisms of $C(E)$. Define $\psi: \operatorname{Aut}(C(E)) \rightarrow H_{E}$ by

$$
\psi: f \mapsto\left(f\left(E_{1}\right) \cdots f\left(E_{n}\right)\right),
$$

where $E_{i}$ denotes the ith column of $E$. Then $\psi$ is an isomorphism of groups. [Dually, the $\mathcal{H}$-class of $E$ is isomorphic to the group of $\mathbb{F} \mathbb{T}$-module automorphisms of $R(E)$.]

Proof. Let $f: C(E) \rightarrow C(E)$ be an $\mathbb{F} \mathbb{T}$-module automorphism and let $A=\psi(f)$. Since $f$ is surjective, it is clear that $C(A)=C(E)$ and hence $A \mathcal{R} E$, by Theorem 3.1 (iii). Now, since $f$ is a linear isomorphism of column spaces, taking the $i$ th column of $E$ to the $i$ th column of $A$, it follows from Theorem 3.3 (ii) that $R(A)=R(E)$, giving $A \mathcal{L} E$ and hence $A \mathcal{H} E$. Thus $\psi$ is well-defined. 
We claim that $\psi$ is a homomorphism of groups. Indeed, let $f, g$ be $\mathbb{F} \mathbb{T}$ module automorphisms of $C(E)$ and let $\psi(f)=A$ and $\psi(g)=B$. Then it is straight-forward to check that $f(x)=A \otimes x$ and $g(x)=B \otimes x$, for all $x \in C(E)$. Moreover, since $B \mathcal{H} E$, we have $B \otimes E=B$ giving

$$
\begin{aligned}
\psi(f \circ g) & =\left(f \circ g\left(E_{1}\right) \cdots f \circ g\left(E_{n}\right)\right) \\
& =\left(A \otimes B \otimes E_{1} \cdots A \otimes B \otimes E_{n}\right) \\
& =A \otimes B \otimes E=A \otimes B=\psi(f) \otimes \psi(g) .
\end{aligned}
$$

In fact, we shall show that $\psi$ is an isomorphism.

We show first that $\psi$ is injective. Indeed, suppose $\psi(f)=\psi(g)$. Then $f\left(E_{i}\right)=g\left(E_{i}\right)$ for all $i$. But the columns $E_{i}$ generate $C(E)$, so by linearity it must be that $f=g$.

It remains to show that this homomorphism is surjective. Let $A \in H_{E}$ and define $f: C(E) \rightarrow C(E)$ by $f(x)=A \otimes x$. Since $H_{E}$ is a group, there exists $A^{\prime} \in H_{E}$ such that $A \otimes A^{\prime}=E=A^{\prime} \otimes A$. Since $E$ acts as the identity on $C(E)$, it follows that $f$ must be bijective; define $f^{\prime}: C(E) \rightarrow C(E)$ by $f^{\prime}(x)=A^{\prime} \otimes x$ and suppose for contradiction that $f(x)=f(y)$ for some $x \neq y$. Then

$x=E \otimes x=A^{\prime} \otimes A \otimes x=f^{\prime}(f(x))=f^{\prime}(f(y))=A^{\prime} \otimes A \otimes y=E \otimes y=y$, contradicting $x \neq y$. Thus $f \in \operatorname{Aut}(C(E))$ and it is clear that $\psi(f)=A$, giving that $\psi$ is surjective.

\section{Dimension, PRojectivity, idempotents And Regularity}

There are several important notions of dimension for a tropical convex set $X \subseteq \mathbb{F T}^{n}$. The tropical dimension is the topological dimension of $X$, viewed as a subset of $\mathbb{R}^{n}$ with the usual topology. Note that, in contrast to the classical (Euclidean) case, tropical convex sets may have regions of different topological dimension. We say that $X$ has pure dimension $k$ if every open (within $\mathrm{X}$ with the induced topology) subset of $\mathrm{X}$ has topological dimension $k$. The generator dimension of $X$ is the minimal cardinality of a generating subset, under the linear operations of scaling and addition. If $X$ is a polytope, this is equal to the number of extremal points of $X$ considered up to scaling $[4,15]$. The dual dimension $[10]$ is the minimal cardinality of a generating set under scaling and the induced operation of greatest lower bound within the convex set. (Notice that, in general, the greatest lower bound of two elements within a convex set $X$ need to be the same as their component-wise minimum, which may not be contained in $X$.)

In [10], the present authors gave a characterisation of projectivity for tropical polytopes in terms of the geometric and order-theoretic structure on these sets. We briefly recall that a module $P$ is called projective if every morphism from $P$ to another module $M$ factors through every surjective module morphism onto $M$. One of the main results of [10] can be summarised as follows.

Theorem 4.1. [10, Theorems 1.1 and 4.5]. Let $X \subseteq \mathbb{F T}^{n}$ be a tropical polytope. Then the following are equivalent:

(i) $X$ is projective as an $\mathbb{F} \mathbb{T}$-module;

(ii) $X$ is the column space of an idempotent matrix in $M_{n}(\mathbb{F} \mathbb{T})$; 
(iii) $X$ has pure dimension equal to its generator dimension and dual dimension.

Since all three notions of dimension coincide for projective polytopes, we define the dimension of a projective tropical polytope to be this common value. We shall refer to projective polytopes of dimension $k$ as projective $k$-polytopes. The following result is a consequence of [10, Theorem 4.2].

Proposition 4.2. [10, Theorem 4.2]. Let $X \subseteq \mathbb{F}^{n}$ be a projective $k$ polytope. Then $X$ is isomorphic to the column space of $a k \times k$ idempotent matrix over $\mathbb{F} \mathbb{T}$.

We note that projective $n$-polytopes in $\mathbb{F}^{n}$ turn out to have a particularly nice structure:

Theorem 4.3. [10, Proposition 5.5]. Let $X \subseteq \mathbb{F T}^{n}$ be a projective $n$ polytope. Then $X$ is min-plus (as well as max-plus) convex.

It is easily verified that any tropical polytope that is min-plus (as well as max-plus) convex must be convex in the usual (Euclidean) sense.

Numerous definitions of rank have been introduced and studied for tropical matrices (see for example $[1,6]$ for more details), mostly corresponding to different notions of "dimension" of the row or column space. In light of Theorem 4.1, we shall focus on the following three definitions of rank. The tropical rank of a matrix is the tropical dimension of its row space (or equivalently, by [7, Theorem 23] for example, its column space). It also has a characterisation in terms of the computation of the matrix permanent [6]. The row rank is the generator dimension of the row space, which by $[10$, Proposition 3.1] is also the dual dimension of the column space. Dually, the column rank is the generator dimension of the column space and also the dual dimension of the row space. Whilst these three notions of rank can in general differ, it follows immediately from Theorem 4.1 that these three ranks must coincide for any idempotent matrix. Thus we shall refer without ambiguity to the rank of an idempotent matrix.

We define a scalar product operation $\mathbb{F}^{n} \times \mathbb{F}^{n} \rightarrow \mathbb{F}^{n}$ on affine tropical $n$-space by setting

$$
\langle x \mid y\rangle=\max \{\lambda \in \mathbb{F} \mathbb{T}: \lambda \otimes x \leq y\} .
$$

This is a residual operation in the sense of residuation theory [2], and has been frequently employed in max-plus algebra. We recall the following result from [13].

Lemma 4.4. $\left[13\right.$, Lemma 5.3] Let $E$ be an idempotent element of $M_{n}(\mathbb{F} \mathbb{T})$. Let $E_{1}, \ldots, E_{n}$ denote the columns of $E$. If $E_{i, i}=0$ then $E_{j, i}=\left\langle E_{j} \mid E_{i}\right\rangle$ for all $j$.

\section{Eigenvalues, EIgenVECTORS AND IDEMPotents}

Let $A \in M_{n}(\mathbb{F} \mathbb{T})$ and let $\Gamma_{A}$ denote the corresponding weighted directed graph. We define the maximum cycle mean of $A$ to be the maximum average ${ }^{5}$

\footnotetext{
${ }^{5} \mathrm{By}$ average, we mean the classical arithmetic mean, which is the tropical geometric mean.
} 
weight of a path from a node to itself in $\Gamma_{A}$. Let $\lambda$ be the maximum cycle mean of $A$. Then the critical graph of $A$ consists of all nodes and edges involved in any path from a node to itself with average weight $\lambda$. The nodes occurring in the critical graph are called critical nodes. It can be shown (see [3], for example) that if $\lambda \leq 0$ then the following series converges to a finite limit, denoted $A^{+}$, in $M_{n}(\mathbb{F} \mathbb{T})$ :

$$
A \oplus A^{2} \oplus \cdots \oplus A^{n} \oplus \cdots .
$$

The following result is well known to experts in tropical mathematics (see [3] for details).

Theorem 5.1. Let $A \in M_{n}(\mathbb{F} \mathbb{T})$ with corresponding weighted directed graph $\Gamma_{A}$. Let $\lambda$ denote the maximum cycle mean of $A$ and set $A_{\lambda}=-\lambda \otimes A$. Then

(i) The maximum cycle mean $\lambda$ is the unique eigenvalue of $A$.

(ii) The columns of $\left(A_{\lambda}\right)^{+}$labelled by the critical nodes form a generating set for the eigenspace of $A$.

(iii) Let $i$ and $j$ be critical nodes. The columns of $\left(A_{\lambda}\right)^{+}$labelled by $i$ and $j$ are tropical multiples of each other if and only if they occur in the same strongly connected component of the critical graph.

(iv) The eigenspace has generator dimension equal to the number of strongly connected components in the critical graph.

From now on let $E$ be an idempotent in $M_{n}(\mathbb{F} \mathbb{T})$. Theorem 5.1 applied to $E$ has a number of interesting consequences.

Corollary 5.2. Let $E \in M_{n}(\mathbb{F} \mathbb{T})$ be an idempotent. Then

(i) $E$ has unique eigenvalue 0 (which is the maximum cycle mean of $E$ ) and corresponding eigenspace $C(E)$.

(ii) $E_{i, i}=0$ if and only if $i$ is a node in the critical graph of $E$.

(iii) The columns of $E$ with diagonal entry 0 form a generating set for the column space of $E$.

(iv) Every extremal point of $C(E)$ occurs up to scaling as a column of $E$ with 0 in the diagonal position.

(v) The rows of $E$ with diagonal entry 0 form a generating set for the row space of $E$.

(vi) Every extremal point of $R(E)$ occurs up to scaling as a row of $E$ with 0 in the diagonal position.

(vii) The rank of $E$ is equal to the number of strongly connected components of the critical graph of $E$.

(viii) Each strongly connected component of the critical graph of $E$ is a complete subgraph of $\Gamma_{E}$.

(ix) If $i$ and $j$ are in the same strongly connected component of the critical graph then the ith column of $E$ is a multiple of the jth column of $E$.

(x) If $i$ and $j$ are in the same strongly connected component of the critical graph then the ith row of $E$ is a multiple of the jth row of $E$.

Proof. (i) Since $E$ is idempotent it is immediate that $E \otimes c=c$ for all columns $c$ of $E$, giving that 0 is an eigenvalue of $E$, with corresponding eigenspace $C(E)$. By Theorem 5.1(i), $E$ has unique eigenvalue equal to the maximum cycle mean of $E$. 
(ii) We note that the critical graph of $E$ consists of all nodes and edges involved in any zero-weighted path from a node to itself. If $E_{i, i}=0$ then, by definition, $i$ is a critical node of $E$. On the other hand, if $i$ is a critical node of $E$ then there is a path from $i$ to $i$ with weight zero. If this path has length $k$, then $E_{i, i}^{\otimes k}=0$. Since $E$ is an idempotent, this gives $E_{i, i}=0$.

(iii) By Theorem 5.1(ii), the columns of $E$ labelled by the critical nodes form a generating set for the eigenspace of $E$. Since the eigenspace of $E$ is equal to the column space of $E$, the result now follows from, part (ii).

(iv) Some scaling of every extremal point must lie in every generating set for $C(E)$.

(vii) Recall that the rank of an idempotent matrix may be defined to be the generator dimension of its column space. The result then follows from Theorem 5.1(iv).

(viii) Let $i$ and $j$ be in the same strongly connected component of the critical graph of $E$ and let $E_{i}$ and $E_{j}$ denote the $i$ th and $j$ th columns of $E$. By Theorem 5.1(iii) we see that $E_{i}=\alpha \otimes E_{j}$ and $E_{j}=-\alpha \otimes E_{i}$. Moreover, since $i$ and $j$ are critical nodes we have $E_{i, i}=E_{j, j}=0$ by part (ii), giving

$$
E_{i, j}+E_{j, i}=\left(\alpha \otimes E_{j, j}\right)+\left(-\alpha \otimes E_{i, i}\right)=\alpha+-\alpha=0 .
$$

Thus we have a zero-weighted path from $i$ to itself via $j$. By definition, this cycle is in the critical graph of $E$. It then follows that each strongly connected component of the critical graph will be a complete graph.

(ix) This follows immediately from Theorem 5.1(iii).

Similar arguments hold for parts (v), (vi) and (x) by considering the row space of $E$ to be the column space of the idempotent $E^{T}$.

Consider the set $C$ of critical nodes of $E$. There is an obvious equivalence relation on $C$, given by $i \sim j$ if and only if $i$ and $j$ are in the same strongly connected component of the critical graph. We call the equivalence classes of this relation the critical classes. Notice that Corollary 5.2 tells us that any set of representatives of the critical classes of $E$ yields a minimal generating set for the row [respectively, column] space of $E$. In fact, by Lemma 3.2, any such set of representatives will give a generating set (not necessarily minimal) for the row [respectively, column] space of any matrix $\mathcal{R}$-below [respectively, $\mathcal{L}$-below] $E$.

Corollary 5.3. Let $A, E \in M_{n}(\mathbb{F} \mathbb{T})$, with $E$ idempotent and let $\left\{c_{1}, \ldots, c_{k}\right\}$ be a set of representatives of the critical classes of $E$.

(i) If $A \leq_{\mathcal{R}} E$ then the rows labelled by $c_{1}, \ldots, c_{k}$ form a generating set for the row space of $A$. If $A \mathcal{R} E$ then this generating set is minimal.

(i) If $A \leq_{\mathcal{L}} E$ then the columns labelled by $c_{1}, \ldots, c_{k}$ form a generating set for the column space of $A$. If $A \mathcal{L} E$ then this generating set is minimal.

Proof. We prove part (i), the proof of part (ii) being dual. It follows immediately from Lemma 3.2 and Corollary 5.2 that the rows labelled by $c_{1}, \ldots, c_{k}$ form a generating set for the row space of $A$. Now suppose that $A \mathcal{R} E$. Then $C(A)=C(E)$ and Theorem 4.1 gives that $C(A)$ has generator dimension equal to its dual dimension. By [10, Proposition 3.1], the dual dimension of $C(A)$ is equal to the generator dimension of $R(A)$. Thus we see that the 
minimum cardinality of a generating set for $R(A)$ is equal to the minimal cardinality of a generating set for $C(A)=C(E)$, which by Corollary 5.2 is equal to $k$.

\section{A Reduction to idempotents of Full RANK}

Let $E \in M_{n}(\mathbb{F} \mathbb{T})$ and suppose that $E$ has rank $k \leq n$. In this section we shall prove that the $\mathcal{H}$-class of $E$, denoted by $H_{E}$, is isomorphic, as a group, to the $\mathcal{H}$-class of a $k \times k$ idempotent $F$ of full rank $k$. We begin by showing that each $\mathcal{D}$-class contains an idempotent whose diagonal entries are all equal to 0 . Since the maximal subgroups in each $\mathcal{D}$-class are all isomorphic, it follows that we may restrict attention to those idempotents with all diagonal entries equal to 0 . Given such an idempotent $E$, the main result of this section (Theorem 6.3) constructs a $k \times k$ idempotent $F$ of full rank $k$ and a group isomorphism between the corresponding $\mathcal{H}$-classes, $H_{E}$ and $H_{F}$. Throughout this section we shall make use of several results and proofs from [10].

Theorem 6.1. Let $E \in M_{n}(\mathbb{F} \mathbb{T})$ be an idempotent. Then the column space of $E$ is min-plus convex if and only if there is an idempotent $F \in M_{n}(\mathbb{F} \mathbb{T})$ such that $F_{i, i}=0$ for all $i$ and $C(F)=C(E)$.

Proof. The statement is trivial for $n=1$. Thus we assume that $n \geq 2$.

Suppose first that $F$ is an idempotent with all diagonal entries equal to zero. We show that $C(F)$ is min-plus convex, using the proof strategy of [10, Proposition 5.5].

Let $x, y \in C(F)$ and let $z$ be the component-wise minimum of $x$ and $y$. It suffices to show that $z \in C(F)$. Since $x, y \in C(F)$ and $F$ is idempotent it is immediate that $x=F \otimes x$ and $y=F \otimes y$. Moreover, since $z \leq x$ and $z \leq y$ we see that $F \otimes z \leq F \otimes x=x$ and $F \otimes z \leq F \otimes y=y$, giving $F \otimes z \leq z$. Using the fact that all the diagonal entries of $F$ are zero yields

$$
(F \otimes z)_{i}=\bigoplus_{j=1}^{n} F_{i, j} \otimes z_{j} \geq F_{i, i} \otimes z_{i}=z_{i},
$$

so that $F \otimes z \geq z$. Thus we have shown that $F \otimes z=z$, giving $z \in C(F)$ as required.

Now suppose that $C(E)$ is min-plus (as well as max-plus) convex. To construct an idempotent $F$ with the desired properties, we use a strategy based on the proof of [10, Theorem 1.4], although the situation here requires us to consider some extra complications. The key idea is to construct a matrix whose $i$ th column is the infimum (in $\mathbb{F}^{n}$ ) of all elements $u \in C(E)$ such that $u_{i} \geq 0$ and show that it has the desired properties. Of course, we must first check that such infima exist.

Let $i \in\{1, \ldots, n\}$ and for each coordinate $j \neq i$, consider the set

$$
\left\{u_{j}: u \in C(E), u_{i} \geq 0\right\} .
$$

It is easy to see that this set is non-empty and, since $C(E)$ is finitely generated, it has a lower bound and hence an infimum. It follows from the fact $C(E)$ is closed that this infimum will be attained. Choose an element 
$w_{j} \in C(E)$ such that $w_{j, j}$ attains this minimum and $w_{j, i} \geq 0$. By the minimality of $w_{j, j}$ and the fact that $C(E)$ is closed under scaling, we must have $w_{j, i}=0$. Now let $F_{i}$ be the minimum of all the $w_{j}$ 's. Then $\left(F_{i}\right)_{i}=0$ and $F_{i}$ is clearly less than or equal to all vectors $u \in C(E)$ with $u_{i} \geq 0$. Thus we have shown that $F_{i}$ is a lower bound for all elements $u \in C(E)$ such that $u_{i} \geq 0$. Suppose that $z$ is another lower bound. By the min-plus convexity of $C(E)$, we see that each $F_{i}$ is itself an element of $C(E)$ with $\left(F_{i}\right)_{i} \geq 0$. Hence $z \leq F_{i}$. Thus $F_{i}$ is the infimum of all elements $u \in C(E)$ such that $u_{i} \geq 0$. Let $F$ be the matrix whose $i$ th column is $F_{i}$. We shall prove that $F$ is an idempotent in $M_{n}(\mathbb{F} \mathbb{T})$ with $C(F)=C(E)$. Since the diagonal entries of $F$ are $F_{i, i}=\left(F_{i}\right)_{i}=0$, this will complete the proof.

We first show that $F$ must be idempotent. It follows from the definition of matrix multiplication that for all $i$ and $j$,

$$
\left(F^{\otimes 2}\right)_{i, j} \geq F_{i, j} \otimes F_{j, j}=F_{i, j}+0=F_{i, j} .
$$

Now let $i, j, k \in\{1, \ldots, n\}$. It will suffice to show that $F_{i, j} \geq F_{i, k} \otimes F_{k, j}$, since then $F_{i, j} \geq \bigoplus_{k=1}^{n} F_{i, k} \otimes F_{k, j}=\left(F^{\otimes 2}\right)_{i, j}$. Consider $w=\left(-F_{k, j}\right) \otimes F_{j}=$ $-\left(F_{j}\right)_{k} \otimes F_{j}$. Then $w \in C(E)$ and $w_{k} \geq 0$. Since $F_{k}$ is the infimum of all such points we have $F_{k} \leq w$. In particular, comparing the $i$ th entries of these elements, we have $\left(-F_{k, j}\right) \otimes F_{i, j}=\left(-F_{k, j}\right) \otimes\left(F_{j}\right)_{i}=w_{i} \geq\left(F_{k}\right)_{i}=F_{i, k}$ and so $F_{i, j} \geq F_{i, k} \otimes F_{k, j}$, as required.

It remains to show that $C(F)=C(E)$. Since each column $F_{i}$ of $F$ is contained in $C(E)$ we have $C(F) \subseteq C(E)$. We shall prove that every extremal point of $C(E)$ occurs, up to scaling, as a column of $F$, so that $C(E) \subseteq C(F)$.

We first show that the columns of $F$ are extremal points of $C(E)$. Suppose for a contradiction that $F_{i}$ is not an extremal point of $C(E)$. Then by definition we may write $F_{i}$ as a finite sum of elements in $C(E)$ which are not multiples of $F_{i}$, say $F_{i}=z_{1} \oplus \cdots \oplus z_{k}$. Let $j$ be such that $0=F_{i, i}=z_{j, i}$. Since $z_{j} \in C(E), z_{j, i} \geq 0$ and $F_{i}$ is the infimum of all such points, we must have $F_{i} \leq z_{j}$. On the other hand, $z_{j}$ forms part of a linear combination for $F_{i}$, giving $z_{j} \leq F_{i}$. So $F_{i}=z_{j}$, contradicting that $z_{j}$ is not a multiple of $F_{i}$.

Now let $x$ be an extremal point of $C(E)$ and let $E_{1}, \ldots, E_{n}$ denote the columns of $E$. By Corollary 5.2 we know that $x$ is a multiple of some column $E_{k}$ with $E_{k, k}=0$. We shall show that $F_{k}=E_{k}$ and hence $x$ occurs up to scaling as a column of $F$, as required. Since $F_{k}$ is an extremal point of $C(E)$, there exists $j$ and $\lambda \in \mathbb{F} \mathbb{T}$ such that $F_{k}=\lambda \otimes E_{j}$ and $E_{j, j}=0$. Moreover, since $F_{k, k}=0$ we find that

$$
0=F_{k, k}=\lambda \otimes\left(E_{j}\right)_{k}=\lambda+E_{k, j},
$$

and hence $\lambda=-E_{k, j}$. On the other hand, $E_{k, k}=0$ and $E_{k} \in C(E)$, so by the definition of $F_{k}$ we have $F_{k} \leq E_{k}$. In other words, $-E_{k, j} \otimes E_{j} \leq E_{k}$. Thus $-E_{k, j} \leq\left\langle E_{j} \mid E_{k}\right\rangle$. Now, since $E_{k, k}=E_{j, j}=0$ we may apply Lemma 4.4 to find $\left\langle E_{j} \mid E_{k}\right\rangle=E_{j, k}$ and $\left\langle E_{k} \mid E_{j}\right\rangle=E_{k, j}$. Thus $E_{k, j}+E_{j, k} \geq 0$. Since $E$ is idempotent, it follows that $-E_{k, j}=E_{j, k}$. Thus $\lambda=-\left\langle E_{k} \mid E_{j}\right\rangle=\left\langle E_{j} \mid E_{k}\right\rangle$ and hence $E_{k}=\lambda \otimes E_{j}=F_{k}$, as required.

Theorem 6.2. Let $E \in M_{n}(\mathbb{F} \mathbb{T})$ be an idempotent. Then there is an idempotent $E^{\prime}$ such that $E \mathcal{D} E^{\prime}$ and $E^{\prime}$ has all diagonal entries equal to 0. 
Proof. Let $E$ be an idempotent of rank $k$. By Proposition 4.2, $C(E) \cong C(F)$ for some $k \times k$ idempotent $F$. It is clear that $F$ must have rank $k$ and hence, by Corollary 5.2 for example, $F$ has all diagonal entries equal to 0 . Moreover, Theorem 4.3 yields that $C(F)$ is min-plus convex. Now let $X$ be the subset of $\mathbb{F T}^{n}$ consisting of all elements $v$ such that: (i) the restriction of $v$ to the first $k$ entries yields an element of $C(F)$ and (ii) all other entries of $v$ are equal to $v_{k}$. It is clear from the definition that $X$ is a tropical polytope which is both max-plus and min-plus convex. Moreover, it is easy to see that $X \cong C(F)$ and hence $X$ is projective. It follows that $X$ is the image of some $n \times n$ idempotent, $X=C\left(E^{\prime}\right)$, say. Theorem 6.1 and the min-plus convexity of $X$ guarantee that $E^{\prime}$ can be chosen with all diagonal entries equal to zero. Hence we have shown that

$$
C(E) \cong C(F) \cong X=C\left(E^{\prime}\right),
$$

where $E, E^{\prime} \in M_{n}(\mathbb{F} \mathbb{T})$. By Theorem 3.1(vi), this gives $E \mathcal{D} E^{\prime}$ as required.

For the rest of this section we shall assume that $E$ is an idempotent matrix in $M_{n}(\mathbb{F} \mathbb{T})$ of rank $k$ whose diagonal entries are all equal to 0 . In the following proof we shall make use of the extended tropical semiring $\mathbb{T}$, defined in Section 2.

Theorem 6.3. Let $E$ be an idempotent matrix in $M_{n}(\mathbb{F} \mathbb{T})$ whose diagonal entries are all equal to 0 . Choose a fixed set of representatives of the critical classes of $E,\left\{c_{1}, \ldots, c_{k}\right\}$ say, and let $M$ be the $k \times n$ matrix with entries in $\mathbb{T}$ defined by $M_{c_{i}, j}=0$ if $j=c_{i}$ and $M_{c_{i}, j}=-\infty$ otherwise. Then

(i) $F=M \otimes E \otimes M^{T}$ is an idempotent of rank $k$ in $M_{k}(\mathbb{F} \mathbb{T})$;

(ii) the map $\phi: A \mapsto M \otimes A \otimes M^{T}$ induces an isomorphism of groups between the $\mathcal{H}$-class of $E$ and the $\mathcal{H}$-class of $F$.

Proof. (i) We first note that, by definition, $F$ is the $k \times k$ submatrix of $E$, whose entries are labelled by $c_{1}, \ldots, c_{k}$. For simplicity, we index the entries of $F$ by $c_{1}, \ldots, c_{k}$ so that $F_{c_{i}, c_{j}}=E_{c_{i}, c_{j}}$. Since each $c_{i}$ is a critical node, it is immediate that all diagonal entries of $F$ are equal to 0 , giving

$$
(F \otimes F)_{c_{i}, c_{j}} \geq F_{c_{i}, c_{i}} \otimes F_{c_{i}, c_{j}}=F_{c_{i}, c_{j}} .
$$

On the other hand, $(F \otimes F)_{c_{i}, c_{j}}$ is equal to the maximum weight of a path of length 2 from node $c_{j}$ to $c_{i}$ via one of $c_{1}, \ldots, c_{k}$. This is clearly bounded above by the maximum weight of a path of length 2 from node $c_{j}$ to $c_{i}$ via any node $1, \ldots, n$. This upper bound is equal to $(E \otimes E)_{c_{i}, c_{j}}$. Since $E$ is idempotent this yields $(F \otimes F)_{c_{i}, c_{j}} \leq(E \otimes E)_{c_{i}, c_{j}}=E_{c_{i}, c_{j}}=F_{c_{i}, c_{j}}$, as required.

(ii) We note that $\phi$ maps each $n \times n$ matrix $A$ to the $k \times k$ submatrix of $A$, with entries labelled by $c_{1}, \ldots, c_{k}$ and, in particular, $F=\phi(E)$. We write $H_{E}$ to denote the $\mathcal{H}$-class of $E$ in $M_{n}(\mathbb{F} \mathbb{T})$ and $H_{F}$ to denote the $\mathcal{H}$ class of $F$ in $M_{k}(\mathbb{F} \mathbb{T})$. Let $A \in H_{E}$. By Corollary 5.3, the columns of $A$ labelled by $c_{1}, \ldots, c_{k}$ form a minimal generating set for the column space of $A$ and the rows of $A$ labelled by $c_{1}, \ldots, c_{k}$ form a minimal generating set for the row space of $A$. This gives $C\left(A M^{T}\right)=C(A)=C(E)$ and $R(M A)=R(A)=R(E)$. Let $\nu: C(E) \rightarrow C(F)$ and $\rho: R(E) \rightarrow R(F)$ be 
the linear maps given by $\nu: v \mapsto M \otimes v$ and $\rho: v \mapsto v \otimes M$. Since $\nu$ maps the columns of $E$ labelled by $c_{1}, \ldots, c_{k}$ to the columns of $F$ labelled by $c_{1}, \ldots, c_{k}$, we see that $\nu$ is onto. Similarly, $\rho$ maps the rows of $E$ labelled by $c_{1}, \ldots, c_{k}$ to the rows of $F$ labelled by $c_{1}, \ldots, c_{k}$, giving that $\rho$ is onto. (In fact, it is straight-forward to check that these maps are isomorphisms of $\mathbb{F} \mathbb{T}$-modules.) It follows that $C(F)=\nu(C(E))=\nu\left(C\left(A M^{T}\right)\right)=C\left(M A M^{T}\right)=C(\phi(A))$ and $R(F)=\rho(R(E))=\rho(R(M A))=R\left(M A M^{T}\right)=R(\phi(A))$. Thus, for all $A \in H_{E}$, we have shown that $\phi(A) \mathcal{H} F$. In other words, $\phi(A) \in H_{F}$ for all $A \in H_{E}$ so that $\phi$ restricts to a map from $H_{E}$ to $H_{F}$. Moreover, it follows from the fact that $\nu$ and $\rho$ are isomorphisms that $\phi$ is injective. We claim that $\phi: H_{E} \rightarrow H_{F}$ is an isomorphism of groups.

Let $A, B \in H_{E}$. We must show that $\phi(A \otimes B)=\phi(A) \otimes \phi(B)$. In other words, we want to show that $M A B M^{T}=M A M^{T} M B M^{T}$ for all $A, B \in H_{E}$. It is straight-forward to verify from the definition of matrix multiplication that this amounts to proving the following claim.

Claim: For every pair $i, j \in\{1, \ldots, k\}$ there exists $t \in\{1, \ldots, k\}$ such that $(A \otimes B)_{c_{i}, c_{j}}=A_{c_{i}, c_{t}} \otimes B_{c_{t}, c_{j}}$.

Suppose for contradiction that that this is not the case. Then there exists $s \notin\left\{c_{1}, \ldots, c_{k}\right\}$ such that

$$
(A \otimes B)_{c_{i}, c_{j}}=A_{c_{i}, s} \otimes B_{s, c_{j}}>A_{c_{i}, c_{l}} \otimes B_{c_{l}, c_{j}},
$$

for all $l \in\{1, \ldots, k\}$. Since $E$ has all diagonal entries equal to 0 , we know that $s$ occurs in the same strongly connected component as $c_{t}$ for some $t \in\{1, \ldots, k\}$. Corollary 5.2 yields that column $s$ of $E$ must be $\alpha$ times column $c_{t}$ of $E$, whilst row $s$ of $E$ must be $-\alpha$ times row $c_{t}$ of $E$, for some $\alpha \in \mathbb{F}$. Since $A$ and $B$ are $\mathcal{H}$-related to $E$, it follows from Lemma 3.2 that we also have column $s$ of $A$ is equal to $\alpha$ times column $c_{t}$ of $A$ and row $s$ of $B$ is equal to $-\alpha$ times row $c_{t}$ of $B$. Thus

$$
(A \otimes B)_{c_{i}, c_{j}}=A_{c_{i}, s} \otimes B_{s, c_{j}}=\alpha \otimes A_{c_{i}, c_{t}} \otimes-\alpha \otimes B_{c_{t}, c_{j}}=A_{c_{i}, c_{t}} \otimes B_{c_{t}, c_{j}},
$$

giving a contradiction. So $\phi$ is a homomorphism of groups.

It remains to show that $\phi$ is surjective. Let $N$ denote the $n \times k$ matrix defined by $N_{s, c_{t}}=0$ if $s=c_{t}, N_{s, c_{t}}=E_{s, c_{t}}$ if $s \notin\left\{c_{1}, \ldots, c_{k}\right\}$ and $N_{s, c_{t}}=$ $-\infty$ otherwise and let $P$ denote the $k \times n$ matrix defined by $P_{c_{t}, s}=0$ if $s=c_{t}, P_{c_{t}, s}=E_{c_{t}, s}$ if $s \notin\left\{c_{1}, \ldots, c_{k}\right\}$ and $P_{c_{t}, s}=-\infty$ otherwise. Notice that

$$
\phi(N K P)=M(N K P) M^{T}=(M N) K\left(P M^{T}\right)=K,
$$

for all $K \in M_{k}(\mathbb{F} \mathbb{T})$. Let $G \in H_{F}$. We claim that $N G P \in H_{E}$, hence giving that $\phi$ is a surjection.

By the definition of $P$, the columns of $N G P$ labelled by $c_{1}, \ldots, c_{k}$ are the columns of $N G$, and all other columns are linear combinations of these. Thus $C(N G P)=C(N G)$. Since $G \in H_{F}$ we have that $F G=G$, giving $C(N G)=C(N F G)$ and it is clear that $C(N F G) \subseteq C(N F)$. Finally, it is straight-forward to check from the definitions of $N$ and $F$ that $N F$ is the $n \times k$ submatrix of $E$ whose columns are labelled by $c_{1}, \ldots, c_{k}$. This gives that $C(N F)=C(E)$ and hence we have shown that $C(N G P) \subseteq C(E)$.

Consider the linear map $\alpha: \mathbb{F} \mathbb{T}^{k} \rightarrow \mathbb{F}^{n}$ given by $v \mapsto N \otimes v$. It is clear from the definition of $N$ that $\alpha$ is one-to-one. Moreover $\alpha$ maps the $c_{i}$ th column of $G$ to the $c_{i}$ th column of $N G P$, inducing a map $\alpha^{\prime}: C(G) \rightarrow$ 
$C(N G P)$. As noted above, the columns of $N G P$ labelled by $c_{1}, \ldots, c_{k}$ form a generating set for $C(N G P)$ and hence $\alpha^{\prime}$ is onto. This shows that $C(G) \cong$ $C(N G P)$ via $\alpha^{\prime}$. Hence we have $C(N G P) \cong C(G)=C(F) \cong C(E)$ and $C(N G P) \subseteq C(E)$. Since $C(N G P), C(E) \subseteq \mathbb{F}^{n}$, the only way this can happen is if $C(N G P)=C(E)$. Thus $N G P \mathcal{R} E$.

Dual arguments will show that $R(N G P) \subseteq R(E)$ and $R(E) \cong R(N G P)$, giving $N G P \mathcal{L} E$ and hence $N G P \mathcal{H} E$, as required.

\section{The $\mathcal{H}$-Class of AN IDEMPOTENT OF FULL RANK}

Let $E$ be an idempotent in $M_{n}(\mathbb{F} T)$ and let $H_{E}$ denote the $\mathcal{H}$-class of $E$. We can consider the matrices in $H_{E}$ as maps from $\mathbb{F} \mathbb{T}^{n}$ to $\mathbb{F}^{n}$, acting by left multiplication, and it follows from the proof of Theorem 3.4 that these maps restrict to $\mathbb{F} \mathbb{T}$-module automorphisms of $C(E)$. We shall show that when $E$ has rank $n$, these automorphisms are affine linear maps, in the classical sense. It then follows that every automorphism of $C(E)$ extends to an automorphism of $\mathbb{F} \mathbb{T}^{n}$.

We note that the boundary of $C(E)$ is the set of all points $y \in C(E)$ for which the equation $E \otimes x=y$ has multiple solutions. Since $E$ acts trivially on $C(E)$, it follows that for every boundary point $y$ there exists some exterior point $z \notin C(E)$ such that $E \otimes z=y$. In fact, it is easy to see that every exterior point must be mapped to the boundary.

Lemma 7.1. [13, Theorem 3.5] Let $E$ be an idempotent of rank $n$ in $M_{n}(\mathbb{F} T)$, and consider the column space $C(E)$ as a subset of $\mathbb{R}^{n}$ equipped with the usual topology. Then left multiplication by $E$ maps all points exterior to $C(E)$ onto the boundary of $C(E)$.

Now let $A \in H_{E}$. Thus $C(A)=C(E)$. Since $E$ has rank $n$, it follows that the columns of $A$ give a minimal generating set for $C(E)$. Since the minimal generating set is unique up to permutation and scaling, we can find a permutation $\sigma$ and scalars $\lambda_{1}, \ldots, \lambda_{n}$ such that $A_{i}=\lambda \otimes E_{\sigma(i)}$ for all $i$. It now follows easily from [3, Corollary 3.1.3], for example, that the equation $A \otimes x=y$ has a unique solution if and only if the equation $E \otimes x=y$ has a unique solution. Thus it is clear that every boundary point $y$ of $C(E)$ is such that the equation $A \otimes x=y$ does not have a unique solution. Since $A$ acts on $C(E)$ by an automorphism, it follows that for every boundary point $y$ there exists some exterior point $z \notin C(E)$ such that $A \otimes z=y$.

Lemma 7.2. Let $E$ be an idempotent of rank $n$ in $M_{n}(\mathbb{F} \mathbb{T})$, and let $A$ be an element in the $\mathcal{H}$-class of $E$. Define $\phi_{A}: \mathbb{F}^{n} \rightarrow \mathbb{F}^{n}$ to be the map given by left multiplication by $A$. Then

(i) $\phi_{A}$ maps interior points of $C(E)$ to interior points;

(ii) $\phi_{A}$ maps boundary points of $C(E)$ to boundary points and

(iii) $\phi_{A}$ maps all points exterior to $C(E)$ onto the boundary of $C(E)$.

[Dually, right multiplication by $A$ induces an $\mathbb{F} \mathbb{T}$-module automorphism of $R(E)$, mapping interior points to interior points and boundary points to boundary points.]

Proof. It is clear that the image of $\phi_{A}$ is $C(E)$ and it follows from the proof of Theorem 3.4 that $\phi_{A}$ restricts to an automorphism of $C(E)$. Since $H_{E}$ is 
a group, there exists $A^{\prime} \in H_{E}$ such that $A A^{\prime}=A^{\prime} A=E$ and hence $\phi_{A}$ and $\phi_{A^{\prime}}$ are mutually inverse on $C(E)$.

(i) Let $x \in C(E)$ be an interior point of the column space. Suppose for contradiction that $\phi_{A}$ maps $x$ to some point $y$ on the boundary of $C(E)$. Then $\phi_{A^{\prime}}$ must map $y$ back to $x$, so that $E x=A^{\prime} A x=A^{\prime} y=x$. Consider the equation $A z=y$. If $x$ is a solution, it is easy to see that $x$ must be the unique solution (since $\phi_{A}$ is an automorphism of $C(E)$, no other element of $C(E)$ can be a solution; if $z \notin C(E)$ were a solution, then $E z=A^{\prime} A z=$ $A^{\prime} y=x$, contradicting Lemma 7.1). However, we have seen that given any point $y$ on the boundary there exists some $z \notin C(A)$ such that $A z=y$.

(ii) It follows immediately that $A$ must map boundary points to boundary points too; if $A$ maps a boundary point $y$ to an interior point $x$, then $A^{\prime}$ must map $x$ back to $y$, contradicting part (i).

(iii) Finally, let $z \in \mathbb{F}^{n}$ with $z \notin C(E)$ and suppose for contradiction that $A$ maps $z$ to an interior point $x$. By Lemma 7.1 we know that $E$ must map $z$ to a point on the boundary, $y$ say. Now $A^{\prime} x=A^{\prime} A z=E z=y$, giving that $A^{\prime}$ maps an interior point, contradicting part (i).

Recall that $\mathbb{T}=\mathbb{F} \mathbb{T} \cup\{-\infty\}$. Let us briefly consider the semigroup $M_{n}(\mathbb{T})$. It is clear that this is a monoid, whose identity element is the $n \times n$ matrix with 0 entries on the diagonal and $-\infty$ entries off the diagonal. It is well known that the units of $M_{n}(\mathbb{T})$ are precisely the tropical monomial matrices, that is, those matrices with exactly one entry not equal to $-\infty$ in each row and in each column. Thus it is clear that every unit has the form $D\left(\lambda_{1}, \ldots, \lambda_{n}\right) P_{\sigma}$, where $D\left(\lambda_{1}, \ldots, \lambda_{n}\right)$ is a diagonal matrix with entries $\lambda_{1}, \ldots, \lambda_{n}$ and $P_{\sigma}$ is a tropical permutation matrix whose $i$ th row has a 0 in the $\sigma(i)$ th position and $-\infty$ entries elsewhere. We shall now show that given an idempotent $E$ of rank $n$ in $M_{n}(\mathbb{F} \mathbb{T})$, the corresponding $\mathcal{H}$-class $H_{E}$ is isomorphic to a certain subgroup of the group of units in $M_{n}(\mathbb{T})$.

Theorem 7.3. Let $E$ be an idempotent of rank $n$ in $M_{n}(\mathbb{F} \mathbb{T})$ and let $H_{E}$ denote the $\mathcal{H}$-class of $E$. Let $G_{E}$ be the set of all units $G \in M_{n}(\mathbb{T})$ which commute with $E$. Then there is a group isomorphism:

$$
G_{E} \rightarrow H_{E}, G \mapsto G E=E G .
$$

Proof. Let $\gamma: G_{E} \rightarrow H_{E}$ be the map defined by $G \mapsto G E=E G$. It is easy to see that $\gamma$ is a homomorphism of groups; indeed, for $G, H \in G_{E}$,

$$
\gamma(G H)=E(G H)=E^{2}(G H)=E(E G) H=(E G)(E H)=\gamma(G) \gamma(H) .
$$

For injectivity, suppose $E G=E H$ for some units $G, H \in G_{E}$. Then $E G H^{-1}=E$. Note that since $G H^{-1}$ is a monomial matrices, each column of $E G H^{-1}$ is a scaling of a permutation of a column of $E$. But since $E$ has rank $n$, no column of $E$ is a scaling of another column. It follows that $G H^{-1}$ must be the identity element, giving that $G=H$.

It remains to show that $\gamma$ is surjective. Let $A \in H_{E}$. By Corollary 5.3, the columns of $A$ provide a minimal generating set for the column space of $E$. It follows that we may choose a unit $G \in M_{n}(\mathbb{T})$ such that $A=E G$. Let $x$ be an interior point of $C(E)$. By Lemma 7.2, $A$ maps $x$ to an interior point. It follows that $G$ must also map $x$ to an interior point; if not then, by Lemma 7.1, $E G$ maps $x$ to the boundary of $C(E)$, contradicting $A=E G$. 
Thus it is easy to see that $G x=A x$ for all interior points $x$. Since $C(E)$ has pure dimension, every boundary point is a limit of interior points. Since $G$ and $A$ are continuous map, it follows that $G x=A x$ for all $x \in C(E)$. In particular, by Theorem 3.4, the action of $G$ restricted to $C(E)$ is an $\mathbb{F} \mathbb{T}$ module automorphism. Now for all $x \in C(E)$ we have $E G x=G x=G E x$, giving

$$
E G=A=A E=E G E=G E E=G E .
$$

Our main theorems combine to prove some results which may be of independent interest.

Theorem 7.4. Every automorphism of a projective n-polytope

(i) extends to an automorphism of $\mathbb{F}^{n}$; and

(ii) is a (classical) affine linear map.

Proof. Let $X \subseteq \mathbb{F}^{n}$ be a projective $n$-polytope. Then by Theorem 4.1, $X=C(E)$ for some full rank idempotent $E \in M_{n}(\mathbb{F} \mathbb{T})$. By Theorem 3.4, there is a matrix $A \in H_{E}$ such that $\phi(x)=A x$ for all $x \in C(E)=X$. By Theorem 7.3 that there is a unit $G \in M_{n}(\mathbb{T})$ such that $A=E G=G E$. Now for any $x \in X=C(E)$ we have

$$
G x=G E x=A x=\phi(x)
$$

so map

$$
\mathbb{F} \mathbb{T}^{n} \rightarrow \mathbb{F}^{n}, x \mapsto G x
$$

is an automorphism of $\mathbb{F} \mathbb{T}^{n}$ extending $\phi$ as required to establish (i).

Now since $G$ is a monomial matrix, we have

$$
G_{i, j}= \begin{cases}\lambda_{i} & \text { if } j=\sigma(i) \\ -\infty & \text { otherwise }\end{cases}
$$

Thus, for all $x \in C(E)$,

$$
(A \otimes x)_{i}=(G E \otimes x)_{i}=(G \otimes x)_{i}=\lambda_{i} \otimes x_{\sigma(i)}=x_{\sigma(i)}+\lambda_{i},
$$

giving $\phi_{A}(x)=P \cdot x+\lambda$, where $P$ is the (classical) permutation matrix corresponding to $\sigma$ and $\lambda=\left(\lambda_{1}, \ldots, \lambda_{n}\right)^{T}$.

Since an $\mathbb{F} \mathbb{T}$-module automorphism $f$ of $C(E)$ respects scaling, it induces a well-defined map $\hat{f}$ on the projectivisation of $C(E)$. It turns out that, in the case $E$ is an idempotent matrix of full rank, this map too is affine linear.

Corollary 7.5. Let $E$ be an idempotent of rank $n$ in $M_{n}(\mathbb{F} \mathbb{T})$, let $A$ be an element in the $\mathcal{H}$-class of $E$ and let $\widehat{\phi_{A}}: \mathcal{P} C(E) \rightarrow \mathcal{P} C(E)$ denote the corresponding map induced by left multiplication by $A$. Then $\widehat{\phi_{A}}$ is a (classical) affine linear map on $\mathcal{P} C(E)$, regarded as a subset of $\mathbb{R}^{n-1}$.

Proof. By Theorem 7.4, $\phi_{A}(x)=P \cdot x+\lambda$, where $P$ is a (classical) permutation matrix and $\lambda=\left(\lambda_{1}, \ldots, \lambda_{n}\right)^{T}$ is a constant vector. Let

$$
P_{i, j}= \begin{cases}1 & \text { if } j=\sigma(i) \\ 0 & \text { otherwise }\end{cases}
$$


for some permutation $\sigma \in S_{n}$. Then

$$
\phi_{A}(x)=\left(\begin{array}{c}
x_{\sigma(1)} \\
\vdots \\
x_{\sigma(n)}
\end{array}\right)+\left(\begin{array}{c}
\lambda_{1} \\
\vdots \\
\lambda_{n}
\end{array}\right)
$$

We may identify $\mathcal{P} \mathbb{F} \mathbb{T}^{(n-1)}$ with $\mathbb{R}^{(n-1)}$ via the map

$$
\left(x_{1}, \ldots, x_{n}\right) \mapsto\left(x_{1}-x_{n}, \ldots, x_{n-1}-x_{n}\right)
$$

and hence

$$
\widehat{\phi_{A}}\left(\begin{array}{c}
x_{1}-x_{n} \\
\vdots \\
x_{n-1}-x_{n}
\end{array}\right)=\left(\begin{array}{c}
x_{\sigma(1)}-x_{\sigma(n)} \\
\vdots \\
x_{\sigma(n-1)}-x_{\sigma(n)}
\end{array}\right)+\left(\begin{array}{c}
\lambda_{1}-\lambda_{n} \\
\vdots \\
\lambda_{n-1}-\lambda_{n}
\end{array}\right) .
$$

If $\sigma(n)=n$ then it is immediate that $\widehat{\phi_{A}}$ is an affine linear map on $\mathcal{P} C(E)$ regarded as a subset of $\mathbb{R}^{n-1}$. Suppose that $\sigma(n) \neq n$. Then $\sigma(k)=n$ for some $k \in\{1, \ldots, n-1\}$. Let $B$ be the $(n-1) \times(n-1)$ matrix given by

$$
B_{i, j}= \begin{cases}1 & \text { if } i \neq k \text { and } j=\sigma(i), \\ -1 & \text { if } j=\sigma(n), \\ 0 & \text { otherwise. }\end{cases}
$$

Then it is easy to check that

$$
\widehat{\phi_{A}}\left(\begin{array}{c}
x_{1}-x_{n} \\
\vdots \\
x_{n-1}-x_{n}
\end{array}\right)=B \cdot\left(\begin{array}{c}
x_{1}-x_{n} \\
\vdots \\
x_{n-1}-x_{n}
\end{array}\right)+\left(\begin{array}{c}
\lambda_{1}-\lambda_{n} \\
\vdots \\
\lambda_{n-1}-\lambda_{n}
\end{array}\right) \text {. }
$$

We shall use Theorem 7.3 to prove our main result; that is, that every maximal subgroup $H_{E}$ is isomorphic to a direct product $\mathbb{R} \times \Sigma$, where $\Sigma$ is a subgroup of $S_{n}$. We shall require the following two lemmas, concerning units that commute with elements of $M_{n}(\mathbb{F} \mathbb{T})$.

Lemma 7.6. Let $A \in M_{n}(\mathbb{F} \mathbb{T})$ and let $G \in M_{n}(\mathbb{T})$ be a unit which commutes with $A$. Then $G$ has only one eigenvalue.

Proof. Suppose false. It is easy to see that some power $G^{k}$ of $G$ is a diagonal matrix. Because $G$ has distinct eigenvalues, so does $G^{k}$, so $G^{k}$ cannot be a scaling matrix. But now it is easy to see that $G^{k}$ cannot commute with any matrix in $M_{n}(\mathbb{F} \mathbb{T})$, which contradicts the assumption that $G$ commutes with $A$.

Lemma 7.7. Let $A \in M_{n}(\mathbb{F} \mathbb{T})$ and let $G$ and $H$ be a units which commute with $A$. Suppose also that $G$ and $H$ have maximal cycle mean 0 . Then $G H$ has maximal cycle mean 0 .

Proof. It follows from the Lemma 7.6 that every cycle in the graphs corresponding to $G$ and $H$ has mean weight 0 . In particular, the (classical) sum of all the finite entries in $G$ is equal to 0 , and the sum of the finite entries in $H$ is equal to 0 . By a simple calculation, the sum of the finite entries in the product $G H$ must also be 0 . Now, since the product $G H$ also commutes 
with $A$, applying Lemma 7.6 again yields that every cycle in $G H$ has the same average weight. Since the sum of the entries (which is 0 ) is a weighted sum of the cycle means, and the cycle means are all the same, we deduce that the cycle means are all 0 . In particular, the maximum cycle mean is 0 .

Theorem 7.8. Let $E$ be an idempotent of rank $n$ in $M_{n}(\mathbb{F} \mathbb{T})$ and let $G_{E}$ denote the group of units commuting with $E$. Define $R=\left\{\lambda \otimes I_{n}: \lambda \in \mathbb{F} \mathbb{T}\right\}$ and $\Sigma=\left\{G \in G_{E}: G\right.$ has eigenvalue 0$\}$. Then $R$ and $\Sigma$ are subgroups of $G_{E}$, and $G_{E} \cong R \times \Sigma$.

Proof. It is easy to see that $R \unlhd G_{E}$. Moreover, the only diagonal matrices which commute with $E$ are those contained in $R$. Since $I_{n}$ has eigenvalue 0 we have $I_{n} \in \Sigma$. Let $A, B \in \Sigma$. By Lemma 7.7 we see that $A B \in \Sigma$ and it is also clear that $A^{-1} \in \Sigma$, giving that $\Sigma \leq G_{E}$. Thus $R, \Sigma \leq H_{E}$ and it is clear that $R \cap \Sigma=\left\{I_{n}\right\}$. Let $G \in G_{E}$. By Lemma 7.6 $G$ has a unique eigenvalue, $\lambda$ say. Hence we may write $G=\lambda \otimes I_{n} \otimes G_{\lambda}$, where $G_{\lambda} \in \Sigma$. In other words, $G_{E}=R \Sigma$. It is clear that every element of $R$ commutes with every element of $\Sigma$, giving $G_{E}=R \times \Sigma \cong \mathbb{R} \times \Sigma$.

It is clear that $R \cong \mathbb{R}$. Moreover, from the definition of matrix multiplication it is easy to show that the map $\phi: G_{E} \rightarrow S_{n}$ given by $D\left(\lambda_{1}, \ldots, \lambda_{n}\right) P_{\sigma} \mapsto$ $\sigma$ is a homomorphism of groups, with kernel given by the set of all diagonal matrices that commute with $E$. Thus $\operatorname{ker} \phi=R$ and hence $\Sigma \cong G_{E} / R \cong$ $\operatorname{Im} \phi \leq S_{n}$.

Corollary 7.9. Let $E$ be an idempotent of rank $n$ in $M_{n}(\mathbb{F} \mathbb{T})$ and let $H_{E}$ denote the $\mathcal{H}$-class of $E$. There exists an element $x \in C(E)$ such that $x$ is an eigenvector for all $A \in H_{E}$.

Proof. Let $S=\{\lambda \otimes E: \lambda \in \mathbb{F} \mathbb{T}\}$ and $R=\left\{\lambda \otimes I_{n}: \lambda \in \mathbb{F} \mathbb{T}\right\}$. Since the map $\gamma: G_{E} \rightarrow H_{E}$ given by $\gamma(G)=E G$ is an isomorphism of groups, we see that $S=\gamma(R)$. Hence $S$ is a normal subgroup of $H_{E}$ which is isomorphic to $\mathbb{R}$ and $H_{E} / S$ is isomorphic to $\operatorname{Im} \phi \leq S_{n}$. It is clear that $S$ acts trivially on $\mathcal{P} C(E)$. Thus the quotient group $H_{E} / S$ acts on $\mathcal{P} C(E)$ by affine linear transformations. Since $H_{E} / S$ is a finite group, we may apply a theorem of Day [5, Theorem 1] to find a fixed point $x \in \mathcal{P} C(E)$ common to all elements of $H_{E} / S$. In other words, $x$ is an eigenvector for all elements of $H_{E}$.

Corollary 7.10. Let $H$ be a maximal subgroup of $M_{n}(\mathbb{F} T)$. Then $H$ is isomorphic to a direct product of the form $\mathbb{R} \times \Sigma$ for some $\Sigma \leq S_{n}$.

\section{REFERENCES}

[1] M. Akian, R. Bapat, and S. Gaubert. Max-plus algebra. In Handbook of Linear Algebra. Chapman and Hall, 2006.

[2] T. S. Blyth and M. F. Janowitz. Residuation theory. Pergamon Press, Oxford, 1972. International Series of Monographs in Pure and Applied Mathematics, Vol. 102.

[3] P. Butkovič. Max-linear systems: theory and algorithms. Springer Monographs in Mathematics. Springer-Verlag London Ltd., London, 2010.

[4] P. Butkovič, H. Schneider, and S. Sergeev. Generators, extremals and bases of max cones. Linear Algebra Appl., 421(2-3):394-406, 2007.

[5] M. M. Day. Fixed-point theorems for compact convex sets. Illinois J. Math., 5:585$590,1961$. 
[6] M. Develin, F. Santos, and B. Sturmfels. On the rank of a tropical matrix. In Combinatorial and computational geometry, volume 52 of Math. Sci. Res. Inst. Publ., pages 213-242. Cambridge Univ. Press, Cambridge, 2005.

[7] M. Develin and B. Sturmfels. Tropical convexity. Doc. Math., 9:1-27 (electronic), 2004.

[8] C. Hollings and M. Kambites. Tropical matrix duality and Green's $\mathcal{D}$ relation. J. London Math. Soc. (to appear).

[9] J. M. Howie. Fundamentals of Semigroup Theory. Clarendon Press, 1995.

[10] Z. Izhakian, M. Johnson, and M. Kambites. Pure dimension and projectivity of tropical polytopes. arXiv:1106.4525 [math.RA], 2011.

[11] M. Johnson and M. Kambites. Green's $\mathcal{J}$-order and the rank of tropical matrices. arXiv:1102.2707v1 [math.RA], 2011.

[12] M. Johnson and M. Kambites. Multiplicative structure of $2 \times 2$ tropical matrices. Linear Algebra Appl., 435:1612-1625, 2011.

[13] M. Johnson and M. Kambites. Idempotent tropical matrices and finite metric spaces. Preprint, 2012.

[14] Y. Shitov. Tropical matrices and group representations. arXiv:1203.1522v1 [math.CO], 2012.

[15] E. Wagneur. Moduloïds and pseudomodules I. Dimension theory. Discrete Math., 98(1):57-73, 1991. 